\title{
Coding Rate and Duration of Vocalizations of the Frog, Xenopus laevis
}

\author{
Erik Zornik and Ayako Yamaguchi \\ Biology Department, University of Utah, Salt Lake City, Utah 84112
}

Vocalizations involve complex rhythmic motor patterns, but the underlying temporal coding mechanisms in the nervous system are poorly understood. Using a recently developed whole-brain preparation from which "fictive" vocalizations are readily elicited in vitro, we investigated the cellular basis of temporal complexity of African clawed frogs (Xenopus laevis). Male advertisement calls contain two alternating components -fast trills $(\sim 300 \mathrm{~ms})$ and slow trills $(\sim 700 \mathrm{~ms})$ that contain clicks repeated at $\sim 60$ and $\sim 30 \mathrm{~Hz}$, respectively. We found that males can alter the duration of fast trills without changing click rates. This finding led us to hypothesize that call rate and duration are regulated by independent mechanisms. We tested this by obtaining whole-cell patch-clamp recordings in the "fictively" calling isolated brain. We discovered a single type of premotor neuron with activity patterns correlated with both the rate and duration of fast trills. These "fast-trill neurons" (FTNs) exhibited long-lasting depolarizations (LLDs) correlated with each fast trill and action potentials that were phase-locked with motor outputneural correlates of call duration and rate, respectively. When depolarized without central pattern generator activation, FTNs produced subthreshold oscillations and action potentials at fast-trill rates, indicating FTN resonance properties are tuned to, and may dictate, the fast-trill rhythm. NMDA receptor (NMDAR) blockade eliminated LLDs in FTNs, and NMDAR activation in synaptically isolated FTNs induced repetitive LLDs. These results suggest FTNs contain an NMDAR-dependent mechanism that may regulate fast-trill duration. We conclude that a single premotor neuron population employs distinct mechanisms to regulate call rate and duration.

\section{Introduction}

Some behaviors, such as speech and courtship display, require millisecond accuracy of motor execution; others, such as breathing and feeding, have less stringent requirements for temporal precision. Vocal behaviors fit into the former category requiring high temporal accuracy with a great diversity of complex temporal patterns across the vertebrate taxa, from fish to humans. Many studies have investigated the anatomical and physiological properties of hindbrain centers in frogs (Schmidt, 1992; Rhodes et al., 2007; Zornik and Kelley, 2008), fishes (Bass et al., 1994; RemageHealey and Bass, 2004), birds (Ashmore et al., 2005; Kubke et al., 2005; Wild et al., 2009), and mammals (Jürgens, 2002; Smotherman et al., 2006; Subramanian and Holstege, 2009). However, the cellular mechanisms underlying vocal rhythms are poorly understood, mainly because neural recordings during vocal production are technically difficult. Here, we addressed the issue in African clawed frogs (Xenopus laevis) using an in vitro whole-brain preparation that can produce fictive vocalizations - trains of compound action potentials (CAPs) in the vocal nerve with temporal profiles that closely resemble natural vocalizations (Fig. 2, com-

\footnotetext{
Received May 21, 2012; revised June 17, 2012; accepted June 29, 2012.

Author contributions: E.Z. and A.Y. designed research; E.Z. performed research; E.Z. analyzed data; E.Z. and A.Y. wrote the paper.

The authors' research and preparation of this manuscript have been supported by National Institute of Neurological Disorders and Stroke Grant R01 NS048834, startup funds provided by University of Utah (A.Y.), and the Grass Foundation (E.Z.). We thank Gary Rose, Matt Wachowiak, and Dawn Blitz and two anonymous reviewers for helpful comments on this manuscript.

Correspondence should be addressed to Erik Zornik, 257 South 1400 East, Salt Lake City, UT 84112. E-mail: ezornik@gmail.com.

DOI:10.1523/JNEUROSCI.2450-12.2012

Copyright $\odot 2012$ the authors $\quad 0270-6474 / 12 / 3212102-13 \$ 15.00 / 0$
}

pare sound recordings in $B$ to nerve recordings in $C, G$, and $E$ ) (Rhodes et al., 2007). Using this preparation, we can not only record neuronal activity during fictive calling but can also characterize synaptic and ionic conductances underlying the observed activity. Understanding the cellular and network mechanisms that mediate the production of vocal patterns promises to elucidate general principles that govern temporally precise motor rhythms.

Male Xenopus laevis produce advertisement calls consisting of sequentially repeating trains of clicks (or trills) —an introductory phase with click rate of $20-40 \mathrm{~Hz}$ (variable duration) followed by a fast trill with $\sim 60 \mathrm{~Hz}$ click rate (lasting $\sim 300 \mathrm{~ms}$ ) and a slow trill with $\sim 30 \mathrm{~Hz}$ click rate (lasting $\sim 700 \mathrm{~ms}$ ). In this study, we discovered that fast-trill duration can be modified without changing click rate. Thus, rate and duration appear to be regulated by independent mechanisms. Here, we investigated the cellular basis of these two temporally distinct outputs of the vocal central pattern generator $(\mathrm{CPG})$.

Previous local field potential (LFP) recordings from the dorsal tegmental area of medulla (DTAM) (a vocal premotor nucleus and homolog of the mammalian parabrachial nucleus) during fictive calling revealed activity correlated with both fast-trill rate and duration (Zornik et al., 2010). Also, NMDA receptor (NMDAR) activation in DTAM is necessary for fast-trill production (Zornik et al., 2010). Because fast-trill duration can be modified without changing click rate, we hypothesized that neurons in DTAM possessed distinct mechanisms that code fast-trill rate and duration. To test this, we developed a blind, whole-cell patchclamp technique to record individual neurons in fictively vocalizing brains. We discovered a single DTAM neuron population 
with activity correlated with both fast-trill rate and duration. Intrinsic properties in these neurons seem to dictate the duration and rate of fast trills. Thus, although trill rate and duration are regulated independently, they may be coded by a single type of premotor neuron.

\section{Materials and Methods}

Animals. Sexually mature X. laevis males ( $n=86$; weight, $41.6 \pm 7.6$ g; length, $6.6 \pm 0.4 \mathrm{~cm}$; mean $\pm \mathrm{SD}$ ) were group-housed in a 40 gallon aquarium, fed twice a week (Xenopus Express), and maintained on a $12 \mathrm{~h}$ light/dark cycle. All animal care procedures were approved by the Animal Care and Use Committee of the University of Utah.

Vocal recordings and analyses. Advertisement calls were recorded using a hydrophone (H2; Aquarian Audio Products) suspended in a plastic $8 \mathrm{~L}$ tank. Sexually mature males $(n=5)$ were housed individually in a tank, and vocalizations were recorded between 6:00 P.M. and 10:00 A.M. using a voice-activated recording system (Syrinx software; John Burt, www.syrinxpc.com). Nine to 20 advertisement calls were sampled from each individual and used for further analyses. Fast-trill duration and fast-trill click rates (average of last six clicks of a fast trill) were measured using Raven software (Cornell University Bioacoustics Laboratory, Ithaca, NY). Regression ANOVA was performed for each individual to determine whether the click rates varied depending on the duration of the fast trill using Statview software.

The isolated brain preparation. Animals were anesthetized with $1.3 \%$ tricaine methane sulfonate (500 $\mu \mathrm{l}$ injected subcutaneously; SigmaAldrich) and decapitated. The skulls where placed in ice-cold saline (in mM: $96 \mathrm{NaCl}, 20 \mathrm{NaHCO}_{3}, 2 \mathrm{CaCl}_{2}, 2 \mathrm{KCl}, 0.5 \mathrm{MgCl}_{2}, 10 \mathrm{HEPES}$, and 11 glucose, $\mathrm{pH} 7.8$ ) oxygenated with $99 \% \mathrm{O}_{2}$. Brains were dissected and placed in a $100 \mathrm{~mm}$ Petri dish lined with silicone elastomer (Sylgard; Dow Corning) filled with oxygenated saline. Immediately after dissection, a complete midline incision was made between the fourth and third ventricles dorsal to the ventricles and aqueduct. After an hour of acclimation to room temperature, brains were transferred to a recording chamber (silicone elastomer-lined $50 \mathrm{~mm}$ Petri dish, $20 \mathrm{ml}$ total volume). Brains were secured to the recording chamber with fine minutien pins (Fine Science Tools) placed in both olfactory nerves and in the spinal cord. Four pins were also placed in the tectum along the midline incision (drawn and pinned laterally) to expose the ventricular surface. Except during drug application (see below), fresh oxygenated saline at room temperature was constantly superfused into the chamber at a rate of at least $150 \mathrm{ml} / \mathrm{h}$.

Electrophysiology. Fictive vocalizations were recorded from the most caudal root of cranial nerve IX-X (N.IX-X) via a suction electrode placed over the nerve. Nerve signals were amplified $(1000 \times)$ and bandpass filtered $(10 \mathrm{~Hz}$ to $10 \mathrm{kHz}$ ) with a differential A-C amplifier (model 1700; A-M Systems). Local field potentials in DTAM were obtained by inserting a $1 \mathrm{M} \Omega$ tungsten electrode (FHC); signals were amplified $(1000 \times)$ and bandpass filtered $(0.1 \mathrm{~Hz}$ to $10 \mathrm{kHz})$ with a microelectrode amplifier (model 1800; A-M Systems).

Whole-cell patch-clamp electrodes were made from thick-walled (1.5 $\mathrm{mm}$ outer diameter; $0.86 \mathrm{~mm}$ inner diameter) borosilicate capillaries with filament (Sutter), fabricated on a Flaming-Brown style P-97 microelectrode puller (Sutter). Electrodes were pulled using a $3 \mathrm{~mm}$ trough filament to have a long taper and relative high resistance (6-10 M $\Omega$ ) to facilitate tissue penetration while limiting tip clogging. Because recordings were performed in the whole isolated brain preparation, a blind search method was used. Positive pressure was applied to the electrode before advancing the electrode (four axis motorized micromanipulator; Siskiyou) through the wall of the fourth ventricle $[\sim 0-300 \mu \mathrm{m}$ ventromedial to the sulcus limitans (Nikundiwe and Nieuwenhuys, 1983); $\sim 100-400 \mu \mathrm{m}$ caudal to the midbrain/cerebellum border]. Cell searches commenced once the electrodes reached a depth of at least $75 \mu \mathrm{m}$; at this point, the rate of electrode advancement was slowed to $\sim 1 \mu \mathrm{m} / \mathrm{s}$. Positive pressure was released once a position was found that produced a rapid, reproducible (verified by retracting at least $10 \mu \mathrm{m}$ and then advancing back to the original position) increase in electrode resistance. Once a gigaohm seal was obtained, whole-cell access was achieved by applying brief negative suction. After obtaining a stable current-clamp recording, positive and negative current steps (2 s) were applied to characterize membrane properties, and then serotonin was applied to characterize cellular activity during fictive vocalizations (see below). In some neurons, voltage-clamp experiments were performed to characterize the synaptic currents received by the neurons during fictive vocalizations. The series resistance was $<15 \mathrm{M} \Omega$ and the series resistance compensation was $90 \%$. Membrane holding potentials were set at either -80 or $-18 \mathrm{mV}$ in an effort to isolate EPSCs and IPSCs, respectively.

Previous anatomical and physiological studies (Zornik and Kelley, 2007,2008 ) have shown a strong excitatory projection from DTAM to n.IX-X. To assess whether DTAM neurons we recorded projected to n.IX-X, we attempted to antidromically activate these neurons by stimulating the motor nucleus, n.IX-X. To do this, we inserted tungsten microelectrodes into n.IX-X and delivered short $(400 \mu \mathrm{s})$ electrical pulses using a Master-8 stimulator via stimulus isolation unit (A.M.P.I.).

Pharmacology. Fictive vocalizations are elicited in the isolated $X$. laevis brain by bath application of serotonin (5-HT) (Sigma-Aldrich) (Rhodes et al., 2007). Final concentrations of 30 or $60 \mu \mathrm{m}$ were obtained by applying $1 \mathrm{ml}$ of concentrated 5-HT stock solution ( 0.6 or $1.2 \mathrm{~mm}$, respectively, in saline) to the recording chamber. We found no difference in either the rate of CAPs or trill duration between the two concentrations, so fictive recordings from the two concentrations were pooled and analyzed in one dataset. To assess whether NMDAR blockade alters membrane potential fluctuations in DTAM neurons, we preincubated brains in $50 \mu \mathrm{M}$ DL-2-amino-5-phosphonopentanoic acid (APV) (SigmaAldrich) in some experiments. To determine whether DTAM neurons expressed NMDARs, we first applied $1 \mu \mathrm{M}$ tetrodotoxin (TTX) (SigmaAldrich) to block all spike-mediated synaptic transmission. The effectiveness of TTX treatment was confirmed when action potentials could no longer be produced by either the FTNs or vocal motoneurons (determined by the loss of activity in the vocal nerve). We then applied $N$-methyl-DL-aspartic acid (NMA) (Sigma-Aldrich; multiple concentrations) (see Results).

Data analysis. We identified a single neuron type with vocal-related activity, which was active mostly during fictive fast trills ["fast-trill neurons" (FTNs)]. FTNs were used for further analysis if their membrane potentials were recorded during at least five fictive advertisement calls. Vocal-related membrane potential data were collected from up to 10 calls (minimum of 5) for each neuron and averaged. Fictive fast trills are often preceded by a variable duration introductory phase. The rate and amplitude of CAPs during introductory phase are lower than those during fast trill, but the transition from introductory phase to fast trill is gradual. In a previous study, we developed a method for objectively defining the onset time of fast trill (Zornik et al., 2010). We first calculated the product of rate and amplitude $\left(R^{\star} A\right)$ for each CAP interval (where the $R$ is the reciprocal of the interval between two consecutive CAPs, and $A$ is the amplitude of the second CAP making up that interval). We then normalized these values $\left(\left[R^{\star} A\right] /\left[\max R^{\star} A\right]^{\star} 100\right.$, where $\max R^{\star} A$ is the maximum $R^{\star} A$ in the same trill), and plotted them in a histogram on a logtransformed $x$-axis. These histograms revealed two peaks with a trough $\sim 50 \%$ of $\max R^{\star} A$. We therefore defined the onset of fast trill as the first CAP interval in a call in which $R^{\star} A$ is $>50 \%$ the $\max R^{\star} A$ for that call. All CAPs in a trill after that interval are defined as fast trill, and all CAPs preceding that time point are designated introductory phase.

We calculated the latency between the FTN action potential and the motor output (CAP) by measuring the interval between the action potential peak and the peak of the subsequent CAP. When a FTN produced doublet or triplet action potentials (see Results), the interval between the first spike and the CAP peak were measured. To examine whether spike to CAP latencies change through the course of a fast trill systematically, we numbered each CAP in reverse order so that CAP number 1 is the last CAP in a fast trill. CAPs were numbered up to 30 (most fast trills did not contain this many CAPs).

Fast-trill onset time was used to calculate the onset time of FTN spiking, normalized to the duration of each introductory phase or fast trill (depending on when the cell began spiking). For neurons that began spiking before or during introductory phase, the "relative onset time" was calculated as spiking onset time relative to a normalized fast-trill 
duration $\left(\left[T_{\mathrm{ftn}}-T_{\text {intro }}\right]\right) / D_{\text {intro }}{ }^{*} 100$, where $T_{\mathrm{ftn}}$ is the FTN spike onset time, $T_{\text {intro }}$ is the time of introductory phase onset, and $D_{\text {intro }}$ is the introductory phase duration). For example, if a FTN begins spiking 10 $\mathrm{ms}$ after the onset of introductory phase, and the total introductory phase duration is $100 \mathrm{~ms}$, the relative onset time for that FTN would be $10 \%$ of the introductory phase. If the FTN began spiking $10 \mathrm{~ms}$ before a $100 \mathrm{~ms}$ introductory phase, the relative onset time would be $-10 \%$ of the introductory phase. A similar equation $\left.\left(\left[T_{\mathrm{ftn}}-T_{\mathrm{fast}}\right]\right) / D_{\mathrm{fast}}{ }^{*} 100\right)$ was performed for FTNs that began spiking during fast trill, where $T_{\text {fast }}$ is the time of fast-trill onset, and $D_{\text {fast }}$ is the duration of the fast trill. The same methods were used to calculate the onset and offset of doublet production by FTNs.

Subthreshold oscillations (STOs) during current step injections were only analyzed if no discernible electrical noise was present before the current steps. STOs were only observed for $\sim 250 \mathrm{~ms}$ in most cases; the limited number of data points ( 2500 data points at $10 \mathrm{kHz}$ sampling rate) did not allow us to use power spectrum analyses to obtain the necessary frequency resolution. Thus, oscillation rates were calculated by measuring 10 interoscillation intervals (during current steps at 100\% rheobase, or during NMA-induced depolarizations), plotting the instantaneous rates (reciprocal of the interval) from each cell in a histogram (bin size, 5 $\mathrm{Hz}$ ), averaging all bins across cells, and then fitting a single-term Gaussian to the resulting data. Rise and decay slopes for STOs during depolarizing current steps (200\% rheobase) were obtained from single events immediately preceding an action potential. To examine the differences in the temporal and amplitude profile of STOs and EPSP, the first EPSP $(>2$ $\mathrm{mV}$ ) associated with a nerve CAP and the last STO (immediately preceding the first action potential) were compared by calculating the slopes for both rise and decay kinetics between 10 and $90 \%$ of total amplitude.

We wanted to analyze spike rates in response to a range of depolarizing current intensities. To do this, we normalized current relative to rheobase and grouped currents into three categories-low (average, $134 \pm$ $2.0 \%$; range, $117-150 \%$ rheobase), medium (average, $166 \pm 1.3 \%$; range, $156-179 \%$ rheobase), and high (average, $199 \pm 0.9 \%$; range, $185-200 \%$ rheobase). We then calculated the instantaneous rates from all spike intervals during a $2 \mathrm{~s}$ current step (in the same manner in which we analyzed STO rates), plotted these in a histogram (bin, $5 \mathrm{~Hz}$ ), and then averaged bin values across cells at each current range. To estimate the most typical spike interval, we then fit a single term Gaussian to each of the three resulting histograms to obtain $\mu \pm \sigma$.

To calculate the rate and duration of rhythmic long-lasting depolarizations (LLDs) induced by NMDAR activation, we calculated the second derivative of the membrane potential; for each LLD, the two largest resulting peaks represented the onset and offset of the LLD. LLD duration was calculated as the time between onset and offset; LLD rate was calculated as the reciprocal of the time from onset of one LLD to the onset of the next. Average values were obtained from 10 consecutive LLDs. Of the 16 FTNs that produced rhythmic LLDs (see Results), 9 produced these at stable patterns for at least $15 \mathrm{~min}$. Neither rate $\left(R^{2}=0.05\right.$; regression ANOVA $\left.F_{(1,7)}=0.351 ; p=0.57\right)$ nor duration $\left(R^{2}=0.13\right.$; regression ANOVA $\left.F_{(1,7)}=1.08 ; p=0.33\right)$ of FTN LLDs differed with the NMA concentrations used $(50-100 \mu \mathrm{M})$. Therefore, the temporal data of the LLDs from these neurons were pooled.

\section{Results}

\section{Fast-trill duration and click rates are} controlled independently

We first examined the relationship between the duration and the rate of fast trills generated by male $X$. laevis. Recordings obtained from five males showed that the fast-trill duration can vary significantly within individuals (Fig. $1 A$ ), but the variation in click rates (measured as the average of the last six clicks of a fast trill) could not be explained by the fast-trill duration in any of the males (regression ANOVA for each of five males: $F_{(1,15)}=0.06$, $p=0.81 ; F_{(1,13)}=2.4, p=0.14 ; F_{(1,12)}=3.4, p=0.09 ; F_{(1,18)}=$ $\left.0.25, p=0.62 ; F_{(1,7)}=2.51, p=0.16\right)$. Thus, we conclude that the click rates remained constant regardless of the fast-trill duration (Fig. $1 B$ ). Fast-trill duration and click rates seem to be controlled
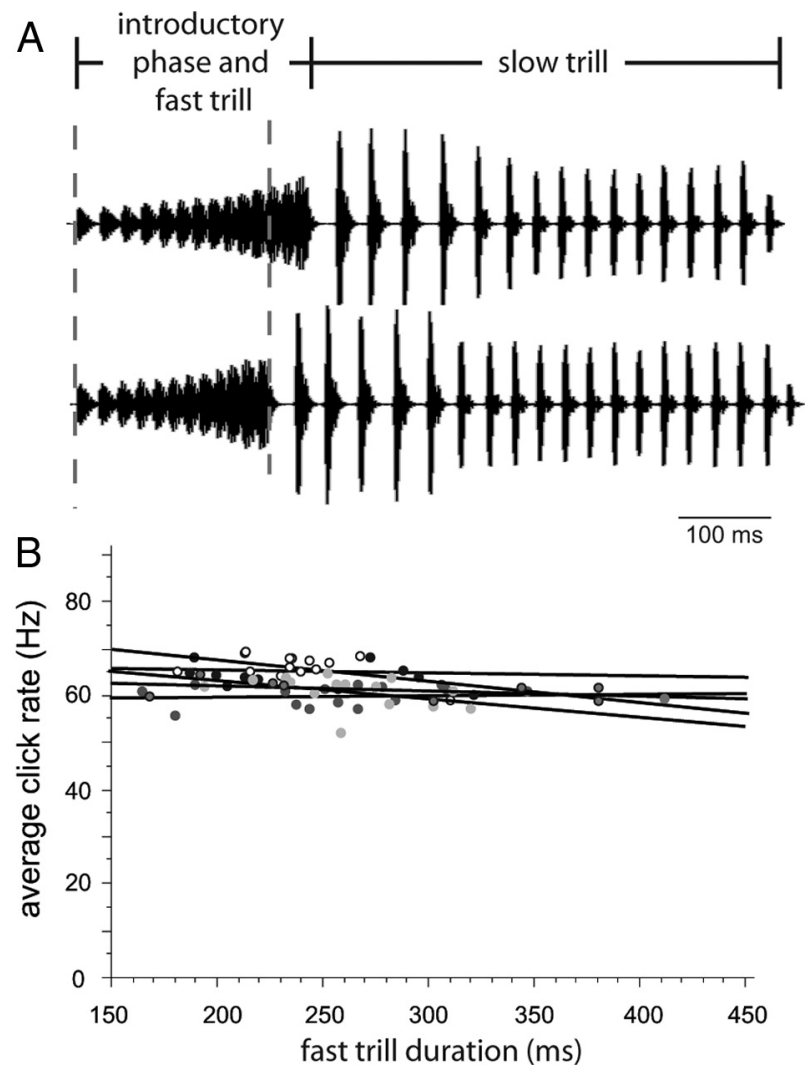

Figure 1. Fast-trill durations vary independently of rate. $A$, Two example sound oscillograms with differing fast-trill durations from a single male. $\boldsymbol{B}$, Regression plots showing fast-trill rates as a function of trill duration for five males (each animal is represented by a unique symbol). Regression ANOVA showed that the variation in click rates is not explained by trill duration. Thus, despite the variation in fast-trill durations, click rates are relatively constant.

independently by the central vocal pathways. The results set the stage to examine how fast-trill duration and click rates are controlled by neurons in the vocal CPG.

\section{Whole-cell recordings in DTAM revealed one predominant cell type}

Previously, we discovered that DTAM is important in controlling both duration and rate of male advertisement calls. Accordingly, we focused on DTAM to understand cellular coding of rhythm and duration. We established a reliable blind patch-clamp method for obtaining robust whole-cell recordings in DTAM of the isolated brain (Fig. 2A), in which we could elicit fictive vocalizations - nerve activity with temporal patterns similar to male advertisement calls produced in vivo (Fig. 2, compare $B$ and top traces of $C, G$, and $E$ ). Because we could maintain quality recordings for many minutes at a time (some recordings lasted an hour or more), we were able to routinely elicit fictive vocalizations while recordings were obtained from these neurons. In total, we obtained whole-cell current-clamp recordings in 126 DTAM neurons during at least one rendition of fictive advertisement calling. Here, we will first describe the variety of neurons we discovered, and then focus on one type of neurons that we found to be important for duration and rate coding during fast trill.

Of all the DTAM neurons from which recordings were obtained during fictive calls, 45\% (57 of 126) did not produce spiking correlated with fictive calls. Most of these produced no action potentials during any phase of fictive calling, although some exhibited slight membrane potential fluctuations correlated vocal 
A

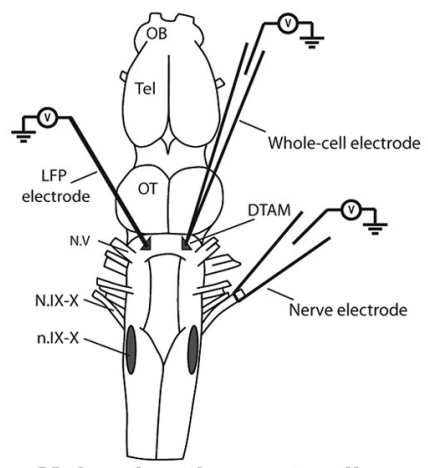

B

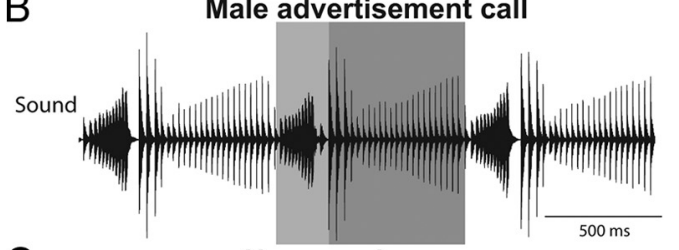

C

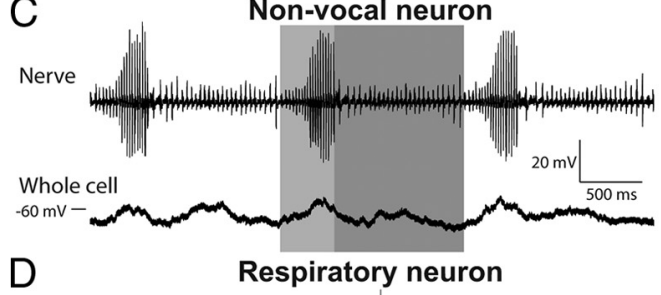

Respiratory neuron
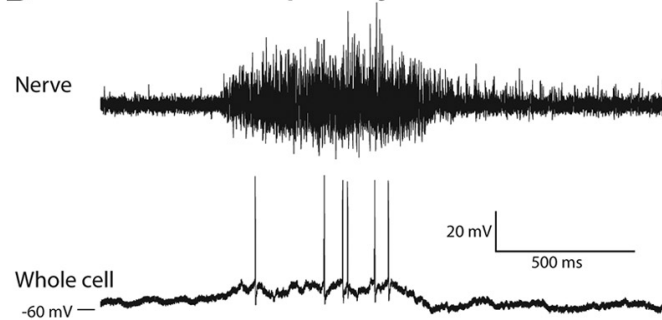

E

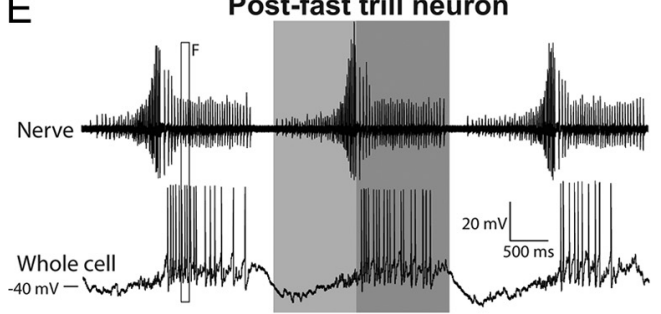

$\mathrm{F}$

Nerve<smiles>C1C2CC1C2</smiles>

Whole cell

$-38 \mathrm{mv}-$

$\mathrm{G}$

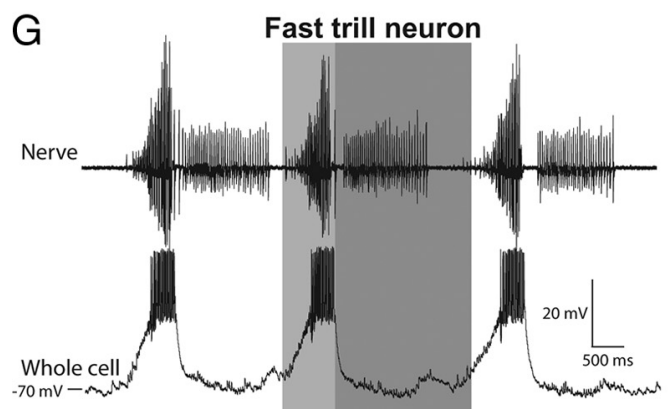

Figure 2. Whole-cell recordings in the isolated brain. $A$, Diagram of an isolated Xenopus laevis brain (dorsal view, anterior is up) showing the two hindbrain CPG nuclei: the DTAM (gray nerve activity (Fig. 2C). Because N.IX-X also contains the axons of glottal motoneurons, we also recorded fictive respiratory nerve activity as has been previously reported (Rhodes et al., 2007; Zornik and Kelley, 2008). A small number of these "nonvocal" DTAM neurons were active during fictive breathing (Fig. 2D).

The remaining $55 \%$ ( 69 of 126) displayed vocal-related action potentials. A small proportion (4\%; 3 of 69 ) of vocal neurons were only active during slow trills or during silent intervals between fast trill and introductory phase (some advertisement calls lack slow trills), but never generated spikes during introductory phase or fast trill (Fig. 2E). Interestingly, the spikes of these "postfast-trill neurons" are not always phase locked to the nerve CAPs; if they are phase locked, the spikes always followed the CAPs (Fig. $2 F$ ). Poor temporal correspondence between the post-fast-trill neuron spikes and CAPs suggest that these neurons do not code for the slow-trill rates. Because of the small number of post-fasttrill neurons recorded so far, we will not discuss these further in this article.

The vast majority of vocal neurons, $96 \%$ (66 of 69), were active during the introductory phase and fast-trill portion of the fictive vocalizations but produced almost no action potentials during slow trills (if present) or during silent intervals between fast trills (Fig. 2G). We named these cells fast-trill neurons to indicate their tendency toward activity during this portion of fictive advertisement calls. FTNs exhibited pronounced LLDs, with overriding action potentials that overlapped with fictive fast trills (Fig. 2G).

Because the FTNs seem to code for both duration and the rate of fast trills, we analyzed the properties of the neurons in detail. Below, we describe (1) how individual FTN spikes correspond to the timing of motor output, (2) how activity of the FTN population relates to the amplitude of motor output, (3) how synaptic inputs and intrinsic FTN properties relate to fast-trill rates, and (4) how FTN LLDs relate to the fast-trill duration.

FTN action potentials are phase-locked to CAPs

FTNs exhibited action potentials that were phase-locked to nerve CAPs during introductory phase and fast trill (Fig. 3). Action potentials either occurred one-to-one ("single spikes"; Fig. 3B), two-to-one ("doublets"; Fig. 3C), or three-to-one ("triplets"; data not shown) with each CAP. Single spikes phase-locked to nerve CAPs were seen in all cells, while some doublets were observed in $\sim 80 \%$ of cells ( 22 of 27 ), and triplets were generated by $\sim 22 \%$ ( 6 of 27 ) of FTNs. Across cells, single spikes accounted for

triangles) in the anterior hindbrain, and the laryngeal motor nucleus, n.IX-X. Motoneuron axons exit the brain via the laryngeal nerve, the caudal root of cranial nerve (N.) IX-X. Fictive vocal activity is recorded by placement of a suction electrode over the nerve. Whole-cell and LFP recordings were performed in DTAM during fictive vocalizations recorded in N.IX-X. B, Example sound oscillogram of three consecutive male advertisement calls showing distinct alternating fast trills (light gray box) and slow trills (dark gray box). C, Example of three consecutive fictive advertisement calls recorded in N.IX-X showing alternating fast and slow trills of CAPs (top trace; compare temporal similarity with sound oscillogram in $\boldsymbol{B}$ ). Whole-cell recording (bottom trace) of a nonvocal neuron that produces no spikes during fictive advertisement calling. $\boldsymbol{D}$, Example of a neuron active during respiratory-related glottal motoneuron firing; these neurons were never active during fictive calling. $\boldsymbol{E}$, Example of three consecutive fictive advertisement calls recorded from the vocal nerve, and a post-fast-trill neuron that only produces action potentials after the fast trills. $\boldsymbol{F}$, Action potentials of a post-fast-trill neuron follow motor CAPs. $\boldsymbol{G}$, Examples of three consecutive fictive advertisement calls (top) recorded in N.IX-X showing alternating fast and slow trills of CAPs. Whole-cell activity (bottom trace) from a DTAM FTN shows a strong depolarization at the onset of the introductory portion of each fast trill, and spiking that mostly occurs during fast trills. Abbreviations: N.V, Trigeminal nerve; N.IX-X, laryngeal nerve; n.IX-X, vocal motor nucleus; OB, olfactory bulb; OT, optic tectum; DTAM, Xenopus parabrachial nucleus; Tel, telencephalon. 
$69 \pm 6 \%$ of CAP-related spiking events, doublets represented $28 \pm 6 \%$, and triplets made up $2 \pm 2 \%$ of CAP-related spiking.

Because some DTAM neurons are known to send direct excitatory projections to vocal motoneurons (Zornik and Kelley, 2008), we suspected that FTNs may provide monosynaptic inputs to drive motor output. We addressed the question of monosynaptic projection in two ways: by antidromically activating FTNs using electrodes inserted into the motor nucleus, and by analyzing the timing between the FTN spikes and CAPs.

We first examined whether FTNs could be antidromically driven by delivering electrical pulses to the laryngeal motor nucleus (n.IX-X) in eight FTNs. In response to brief pulses delivered to n.IX-X, we observed short-latency $(0.4-1.7 \mathrm{~ms})$ antidromic spikes in 75\% (6 of 8 ) of FTNs (see example in Fig. 3D). The fact that n.IX-X stimulation did not evoke antidromic spikes in a subset of FTNs may indicate either that some of these neurons do not project to n.IX-X, or that our stimulating electrode was not always sufficient to antidromically activate all of the incoming FTN axons. In either case, the results confirmed that at least some FTNs project to the motor nucleus.

We next analyzed the timing of the FTNs and CAPs. A previous study (Zornik and Kelley, 2008) demonstrated that a single stimulus pulse delivered to DTAM can elicit monosynaptically driven motor output with $\sim 10$ ms of delay, whereas a train of pulses delivered at $20 \mathrm{~Hz}$ elicits motor output with progressively shorter delays ( $\sim 7 \mathrm{~ms}$ ), either due to presynaptic facilitation, postsynaptic temporal summation, or both (Zornik and Kelley, 2008). We predicted that if FTNs directly project to motoneurons, we should see constant latency between the FTN spikes and the motor CAPs in a similar range. Because fast-trill offset is a stereotyped and salient event identifiable in all calls, we numbered fast-trill CAPs in reverse order, such that CAP number 1 is the final fast-trill CAP and number 30 is the 30 th from last

(Fig. $3 E$ ). With this organization, we could address not only whether the spike-to-CAP latencies are similar to those observed in the previous study mentioned above (Zornik and Kelley, 2008) but also whether spike-to-CAP latencies varied systematically within a call. During the final 15 CAPs when click repetition rate is the highest, we found that latencies from FTN spikes to CAPs varied little, ranging from $\sim 3-4 \mathrm{~ms}$ (Fig. $3 E$ ). Earlier in calls when the CAP repetition rate is slower, however, the latencies were somewhat larger (up to $\sim 8$ $\mathrm{ms})$. Together, we demonstrated that majority of the FTNs project to the motor nucleus, and the latencies observed in this
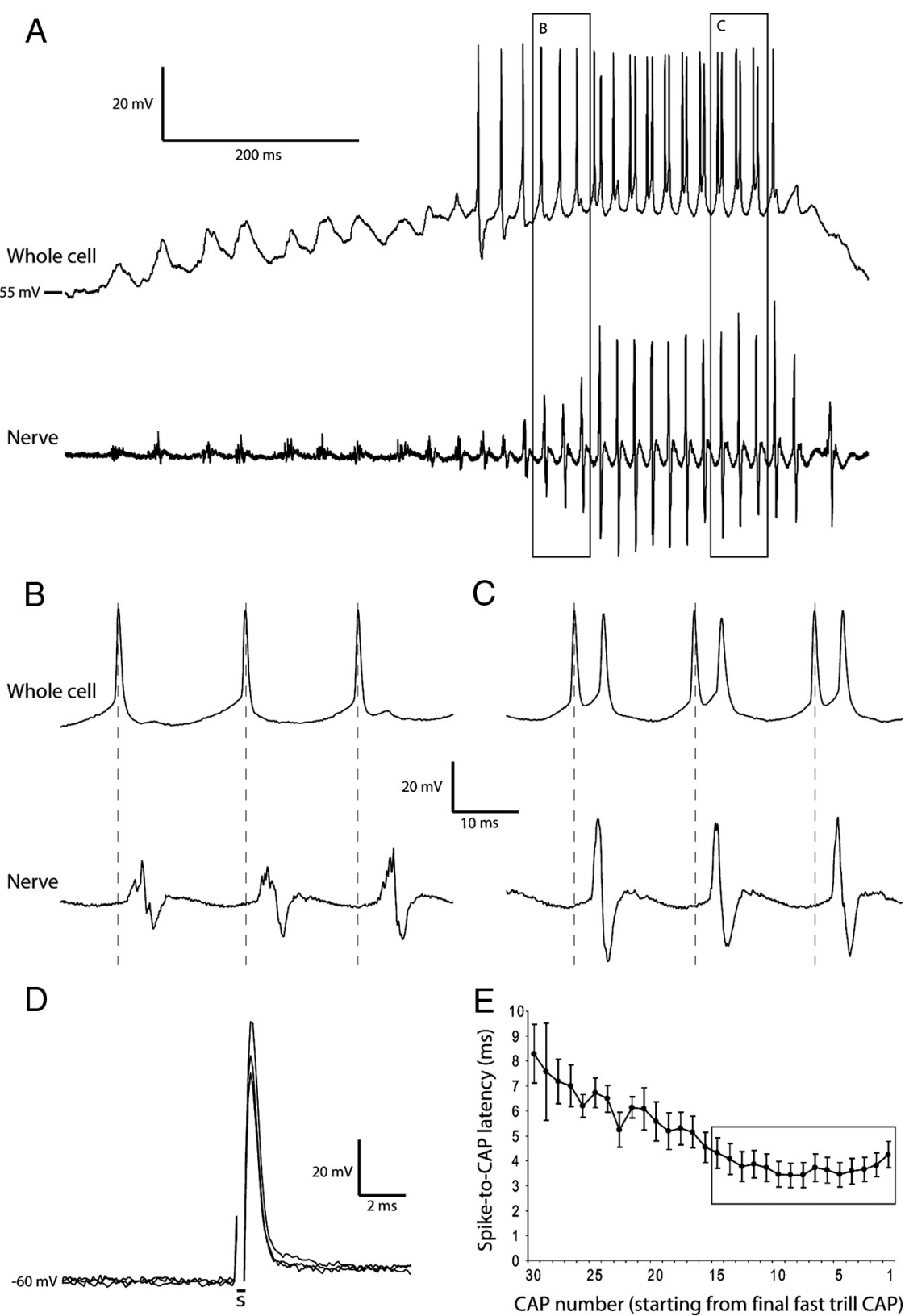

Figure 3. Spike timing of a FTN relative to individual CAPs in the vocal nerve. $\boldsymbol{A}$, Example FTN membrane potential recording (top trace) during one rendition of fictive fast trill (bottom trace). The boxes outline expanded traces shown in $\boldsymbol{B}$ and $\boldsymbol{C}$. $\boldsymbol{B}$, During the early portion of fast trill in this example, the FTN produces a single spike (top) that precedes each nerve CAP (bottom). C, Later in this example, the FTN produces spike doublets phase-locked with each CAP. D, Three overlaid examples of antidromic spikes in a FTN occurring $<1 \mathrm{~ms}$ after stimulating $n$.IX-X. A horizontal bar labeled with "s" indicates the time at which stimulus pulse was delivered to the n.IX-X.E, Plot showing the average latency from the first (or only) action potential to its corresponding CAP over the last 30 CAPs of a fast trill ( 1 being the final CAP of a fast trill). Note that the latencies earlier in a fast trill ( $\sim 8 \mathrm{~ms}$ ) are slightly longer than at the end of a fast trill ( $\sim 4 \mathrm{~ms}$; gray box).

study are consistent with the time frame of monosynaptically driven motor activity. These results suggest that FTN spikes may act as a premotor signal that drives motoneurons monosynaptically.

\section{Coding amplitude by a population of FTNs}

A defining feature of FTNs is that they spiked almost exclusively during fictive introductory phase and fast trill (Figs. 2G, 4). Once FTNs start firing, the activity continued until the end of the fast trill (Fig. 2G,4). Interestingly, the onset of spike trains varied across the population (Fig. $4 A-D$ ), ranging from tens of millisec- 


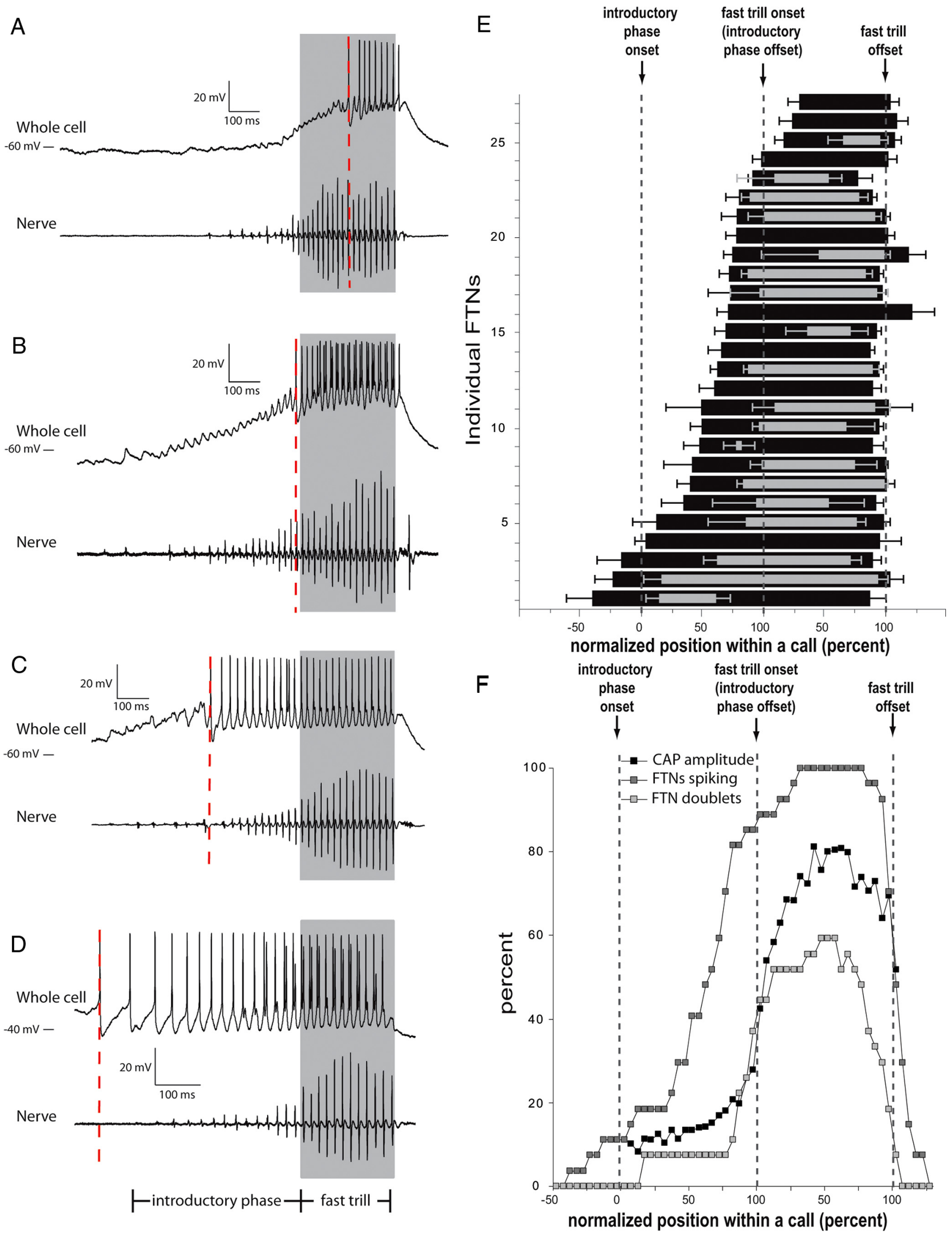

Figure 4. Each FTN is active during distinct portions of introductory phase and fast trill. $\boldsymbol{A}$, Example FTN that produces action potentials during the second half of each fast trill (in each panel: spiking onset highlighted with dotted red line; fast trill designated by gray box; see Materials and Methods for calculation of fast-trill onset). $\boldsymbol{B}$, FTN that begins spiking immediately before fast-trill onset. C, Membrane potential of a FTN that begins spiking during the introductory phase ( $\sim 200 \mathrm{~ms}$ before fast-trill onset). $\boldsymbol{D}$, Activity of a FTN that begins spiking (Figure legend continues.) 
onds before the first CAP of the introductory phase (Fig. $4 D$ ) to the middle of fast trill (Fig. 4A). Despite this variability across cells, the time of activation relative to the call onset was stereotyped within each cell; over many advertisement calls, each neuron began spiking with little variation relative to the position within each call (Fig. 4E). Likewise, doublets were produced at a fairly stereotyped portion of each introductory phase or fast trill in each FTN, but the pattern varied significantly across the population (Fig. 4E). Although doublets and triplets tended to occur during the later portions of fast trills (see example in Fig. 3), there was no significant difference in the average CAP rates associated with single, doublet, and triplet spikes (43.8 $\pm 1.6,46.8 \pm 1.4$, and $39.6 \pm 3.6 \mathrm{~Hz}$, respectively; $H=4.497, p=0.11$, KruskalWallis test), indicating that the number of spikes generated by each FTN per cycle is not related to the CAP rate. Instead, the presence of doublets seems to be related to the CAP amplitude (see below).

Analysis of spike and doublet onset timing of 27 neurons (from which recordings were obtained during five or more fictive advertisement calls) (see Materials and Methods) revealed a progressive recruitment pattern of FTNs (Fig. 4E). By normalizing onset and offset of spike and doublet production relative to fictive nerve activity, we calculated a proportion of active neurons during each phase of a normalized advertisement call. According to our estimate, the percentage of spiking FTNs increases throughout the introductory phase, reaching $100 \%$ participation during the final two-thirds of each fast trill. A similar pattern is observed for FTN doublet production, in which the majority of FTN doublets occur during fast trill (Fig. $4 E, F$ ). Interestingly, this pattern of progressive FTN recruitment closely parallels the pattern of increasing CAP amplitude (Fig. 4F). Correlation coefficients between normalized CAP amplitude and percentage of spiking FTNs was high, 0.895 (Fisher's $r$ to $z, p<0.0001$ ). The correlation coefficients between CAP amplitude and the percentage of FTNs producing doublets was even higher $(r=0.959 ; p<0.0001)$. One possible interpretation of these results is that an increase in the number of active FTNs, especially with doublets, causes increased recruitment of downstream motoneurons, thus generating higher amplitude CAPs associated with the final stage of fast trills.

\section{Coding fictive fast-trill CAP rates: synaptic versus intrinsic mechanisms}

In FTNs that did not spike at the start of the introductory phase, subthreshold activity was observed that was phase-locked with CAPs (Fig. 5A, blue box). Initially, these events resemble EPSPs (rapid onset followed by slower, exponential decay) that temporally summate to gradually depolarize the membrane, leading to

\footnotetext{
(Figure legend continued.) shortly before the first introductory-phase CAP. E, Each horizontal bar in this plot shows the relative spike timing (black bars) or doublet production (gray bars) for 1 of 27 neurons. For each cell, the left edge of a bar represents the mean onset time of spiking or doublet production; the right edge of each bar represents the offset of spiking or doublet activity. Error bars represent the SD of the mean. Note that the SD is relatively low for most cells, indicating that timing of FTN activity relative to a particular phase of a call is stereotyped within each neuron. Also note that most FTNs begin spiking during the introductory phase. All cells are active during the majority of each fast trill. $\boldsymbol{F}$, For each section of a normalized introductory phase and fast trill (bins, 5\%), this plot shows (1) the average normalized CAP amplitude (black squares), (2) the percentage of spiking FTNs (dark gray squares), and (3) the percentage of FTNs producing doublets (light gray squares). Both the percentage of spiking FTNs and the percentage of FTNs generating doublets are strongly correlated with call amplitude, indicating that CAP amplitude may be regulated by the number of active FTNs and the number of spikes produced by each FTN.
}

the onset of the LLDs associated with fast trill. However, as the calls progressed, the kinetics of the subthreshold events riding on top of the slow depolarization changed. Both the rise and decay slopes of the first subthreshold event (Fig. $5 A, B$, in blue) were lower in magnitude (rise, $0.51 \pm 0.04 \mathrm{mV} / \mathrm{ms}$; decay, $-0.31 \pm$ $0.07 \mathrm{mV} / \mathrm{ms}$ ) compared with the last subthreshold event preceding the first spike (Fig. $5 A, B$, in red; rise: $0.84 \pm 0.05 \mathrm{mV} / \mathrm{ms}$; decay: $-1.55 \pm 0.15 \mathrm{mV} / \mathrm{ms} ; n=17$; rise: $U=32, z=-3.875$, $p=0.0001$; decay: $U=7, z=-4.736, p<0.0001$, MannWhitney). One possible explanation for this change in kinetics of the subthreshold event is that, as initial EPSPs lead to depolarization of the FTN, voltage-dependent currents may become activated, which in turn generate the subthreshold oscillation. The results above indicate the existence of intricate interactions between synaptic inputs and intrinsic properties of FTNs during introductory and fast-trill phases. The observation led us to isolate the synaptic activity from intrinsic mechanisms.

To examine the nature of the synaptic inputs received by the FTNs during fictive vocalizations, voltage-clamp experiments were performed. When the membrane potential was held at -18 $\mathrm{mV}$ (the membrane potential could not be brought to the $E_{\mathrm{Na}}$ of $64 \mathrm{mV}$ because the neuron could not be sufficiently space clamped), IPSCs phase-locked to the motor CAPs were recorded (Fig. $5 C, D$ ). When the membrane potential was held at $-80 \mathrm{mV}$ near reversal potential for chloride $\left(E_{\mathrm{Cl}}=-82 \mathrm{mV}\right)$, EPSCs phase-locked to the motor CAPs were recorded (Fig. $5 E, F$ ). Interestingly, the onset of the PSCs were either coinciding with or following the motor CAPs (Fig. 5D,F). Considering that spikes of FTNs precede motor CAPs by 3-8 ms during fictive vocalizations (Fig. 3D), the results of the voltage-clamp experiments suggest that synaptic inputs received by the FTNs do not drive the neurons to fire action potentials. One possible alternative function of the rhythmic synaptic inputs may be to entrain the STOs of the FTNs, possibly via efferent motor copy, thus ensuring synchrony across the FTN population.

Having identified the synaptic inputs received by the FTNs, we next turned our attention to characterizing the intrinsic properties of the STOs generated by the FTNs. To investigate the nature of the STO in the absence of synaptic inputs, we injected depolarizing current steps in FTNs to activate voltage-sensitive currents in the absence of call production. We reasoned that, if the STO is generated by voltage-dependent conductances intrinsic to the FTNs, depolarizing the FTNs should trigger STOs. We found that suprathreshold depolarizing current steps elicited spike bursts preceded by (and sometimes interspersed with) STOs (Fig. $6 A)$. STOs were observed only at membrane potentials near spike threshold (Fig. 6A,B). Accordingly, we were unable to assess whether STO frequency is voltage dependent. When the rates of STOs during rheobase level current steps were measured, we found that STOs were reliably produced at $\sim 60 \mathrm{~Hz}(59.4 \pm 6.7$ $\mathrm{Hz} ; \mu \pm \sigma$; Fig. $6 C$ ). The results suggest that, when depolarized, FTNs express voltage-dependent ionic conductances that generate STOs in a range overlapping with normal fast-trill rates. It is important to note that STOs were not observed in any other types of DTAM neurons including nonvocal and post-fast-trill neurons. This suggests that these intrinsic properties of FTNs may represent a cellular mechanism that contributes to determination of the fast-trill rhythm.

How do STOs generated during call production differ from those elicited in response to current injection? To address this question, we compared the temporal profiles of intrinsic STOs elicited in response to current injection with those produced during fictive vocalizations. The rise slopes during current injections 

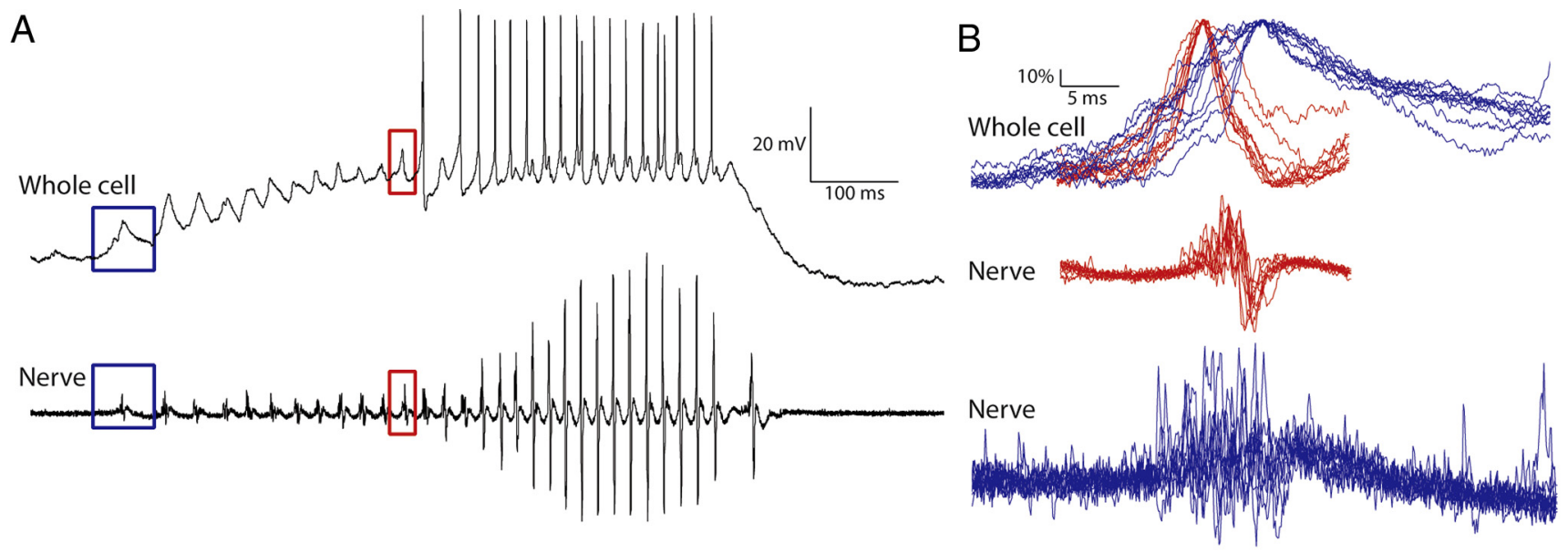

C
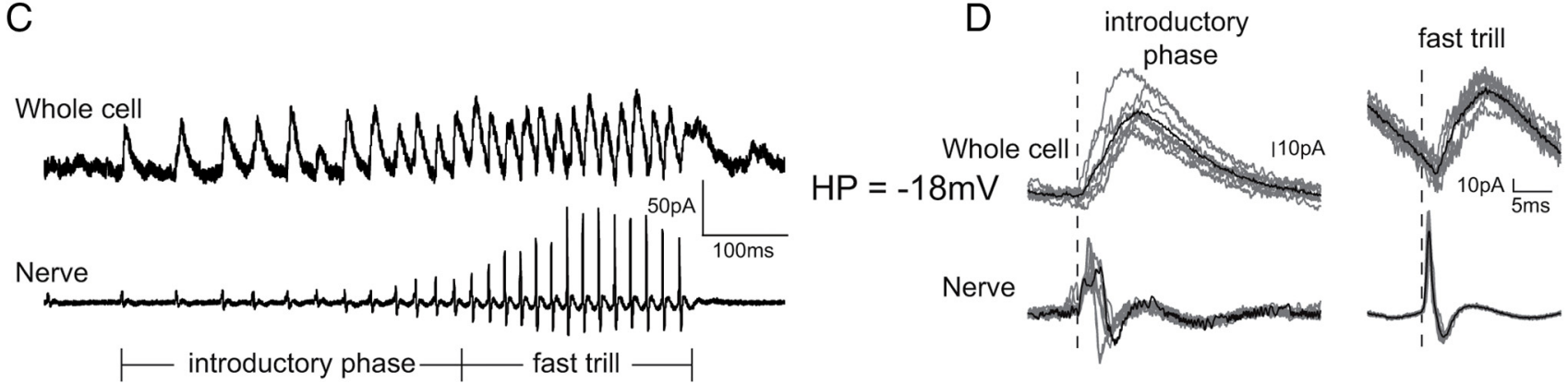

E
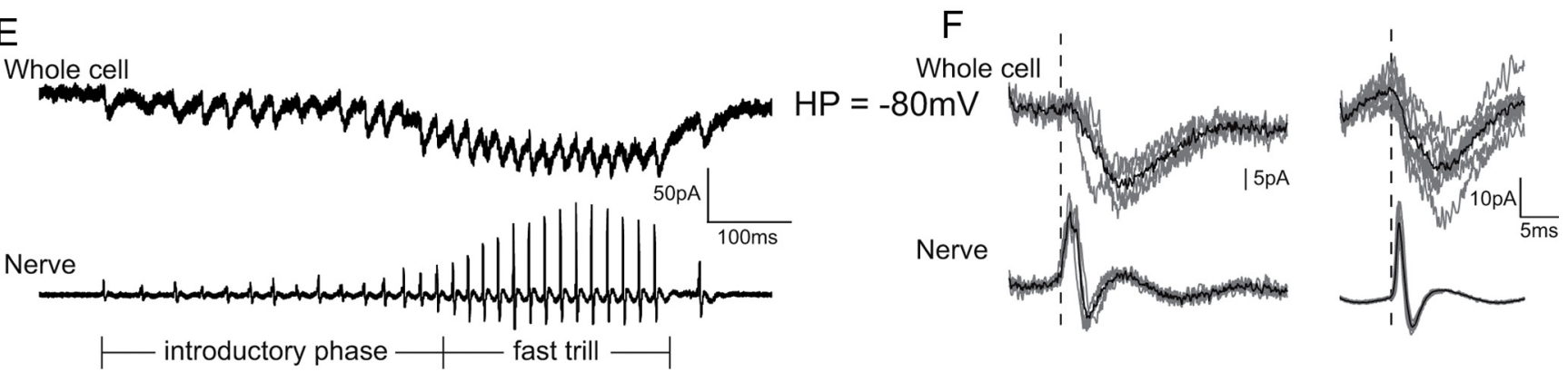

Figure 5. FTNs exhibit two types of subthreshold activity patterns. $\boldsymbol{A}$, Example FTN membrane potential recording (top trace) during one rendition of fictive fast trill (nerve recording; bottom trace). The blue and red boxes outline types of activity shown in $\boldsymbol{B}$. $\boldsymbol{B}$, Example of the first subthreshold potential from 10 different fast trills (top blue traces) show different onset and offset kinetics than subthreshold potentials immediately preceding the first FTN spikes (top red traces). We suggest that the first events (blue) represent EPSPs, while the latter events (red) represent STOs. The two bottom traces in blue and red illustrate the timing of motor nerve activities associated with the EPSPs and STOs. C, Example voltage-clamp recording of a FTN with holding potential (HP) of $-18 \mathrm{mV}$ (top trace) showing IPSCs phase locked with nerve CAPs during a single rendition of introductory phase and fast trill (bottom trace). D, Ten overlaid IPSC traces (top) and nerve CAPs (bottom) during introductory phase and fast trill show that IPSCs tend to follow nerve activity. $\boldsymbol{E}$, Voltage-clamp recording of the same neuron in $\boldsymbol{C}$ and $\boldsymbol{D}$ held at $-80 \mathrm{mV}$ (top trace) during a single rendition of introductory phase and fast trill (bottom trace). Note the downward EPSCs that are phase-locked with nerve activity. $F$, Ten overlaid EPSC traces (top) and nerve CAPs (bottom) show that EPSC onset tends to follow nerve CAPs both during introductory phase and fast trill.

$(0.68 \pm 0.06 \mathrm{mV} / \mathrm{ms} ; n=8)$ were not significantly different from those during fictive fast trills $(0.84 \pm 0.05 \mathrm{mV} / \mathrm{ms} ; n=17 ; U=$ $41, p=0.1157$, Mann-Whitney). However, the slopes of STO decay $(-0.62 \pm 0.08 \mathrm{mV} / \mathrm{ms} ; n=8)$ in response to current steps were significantly slower than STO decay slopes during fictive calling $(-1.55 \pm 0.15 ; n=17 ; U=6, p=0.0003$, MannWhitney). This suggests that synaptic conductances available during fictive calling may modify the decay time of the STO, perhaps by temporarily increasing the membrane conductances during the falling phase of STO.

We next wanted to determine how STOs relate to FTN firing rates. To this end, we analyzed the FTN spike rates in response to three ranges of depolarizing current intensities: low (range, 117$150 \%$ rheobase; mean \pm SE, $134 \pm 2.0 \% ; n=21)$, medium (range, 156-179\%; mean \pm SE, $166 \pm 1.3 \% ; n=19$ ), and high (range, $186-200 \%$; mean \pm SE, $199 \pm 0.9 \%$; $n=11$ ). We measured all spike intervals during a single $2 \mathrm{~s}$ current step (repeated at each current range if possible) and plotted the resulting instantaneous spike rates (reciprocal of the interval) in a histogram (bin, $5 \mathrm{~Hz}$ ), and then averaged each bin across all cells analyzed for each range of currents. Interestingly, resulting histograms showed prominent, tightly clustered peaks near $60 \mathrm{~Hz}$ for all three current ranges (Fig. 6D). Means of the Gaussian curve fitted to each histogram were $58.8 \pm 4.3,59.7 \pm 5.4$, and $60.6 \pm 5.6 \mathrm{~Hz}$ for low, medium, and high current stimulation, respectively $(\mu \pm$ $\sigma)$, indicating that the FTNs have a strong tendency to fire at 60 $\mathrm{Hz}$ regardless of the amount of current injected. Interestingly, histograms obtained from low and medium current data showed a second peak at $30 \mathrm{~Hz}$. This second peak suggests that, when a FTN does not reach the spike threshold, then it has a tendency to 

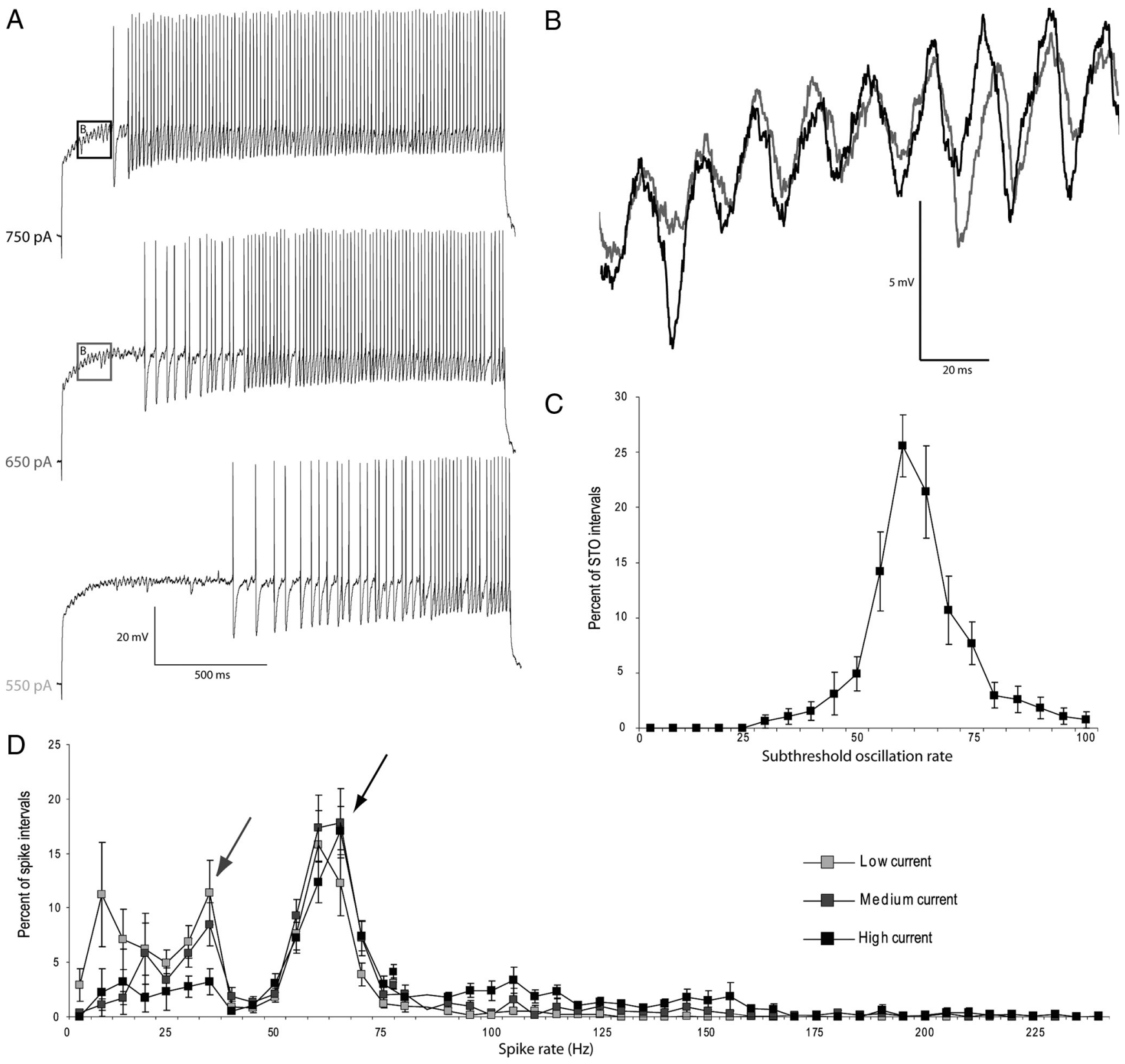

Figure 6. FTNs intrinsically generate fast-trill rhythms. $A$, Three example current injections of $750 \mathrm{pA}$ (top trace), $650 \mathrm{pA}$ (middle trace), and $550 \mathrm{pA}$ (bottom trace) from a single FTN. The dark and light gray boxes highlight the subthreshold activity shown in B. B, STO sare apparent in FTNs during depolarizing current injections. Note the similarity in membrane potentials for two distinct current levels. $\boldsymbol{C}$, The average distributions of STO rates (in response to rheobase level currents) peaks around fast-trill rates $(\sim 60 \mathrm{~Hz})$. $\boldsymbol{D}$, The average distributions of spike rates during injections of low $(134 \pm 2.0 \%$ rheobase; $n=21)$, medium $(166 \pm 1.3 \%$ rheobase; $n=19)$, and high $(199 \pm 0.9 \%$ rheobase; $n=11)$ currents. Note that the largest peak in all cases is $\sim 60 \mathrm{~Hz}($ black arrow); medium and low currents show a secondary peak $\sim 30 \mathrm{~Hz}$ (gray arrow).

wait for another STO interval ( $\sim 16-17 \mathrm{~ms})$ before it spikes again. This indicates the presence of an internal oscillatory clock that increases the probability of firing around the STO rate of $\sim 60 \mathrm{~Hz}$. Together, we conclude that FTNs exhibit intrinsic ionic conductances that promote both subthreshold and suprathreshold oscillatory activity near $60 \mathrm{~Hz}$, indicating that FTNs may be the source of fast-trill rhythm. Rhythmic synaptic inputs received by FTNs that are phase-locked to the motor output may play a role in synchronizing intrinsic oscillatory activity across the FTN population.

\section{FTNs express NMDARs and produce}

\section{NMDAR-dependent LLDs}

FTNs exhibited distinct LLDs (mean \pm SD, $940 \pm 373 \mathrm{~ms}$ ) that coincided with the onset and offset of fast trills (Fig. 7). Ac- cordingly, the total LLD durations correlated significantly with total fast-trill duration $\left(R^{2}=0.62\right.$; regression ANOVA $\left.F_{(1,24)}=16.2, p<0.0005\right)$. In a previous study (Zornik et al., 2010), we showed that LFP recordings obtained from DTAM contain a slow baseline wave that correlated with onset and offset of fast trills (see example in Fig. 7). The results suggest that the summed LLDs across the FTN population may represent the cellular basis for the LFP wave recorded from DTAM, raising the possibility that FTNs control fast-trill duration in addition to the fast-trill rates. Given that NMDAR activation in DTAM is required for fast trill and LFP wave generation (Zornik et al., 2010), and FTNs may be the cellular basis of the wave, we predicted that FTNs may express the NMDARs that underlie generation of LLDs. 


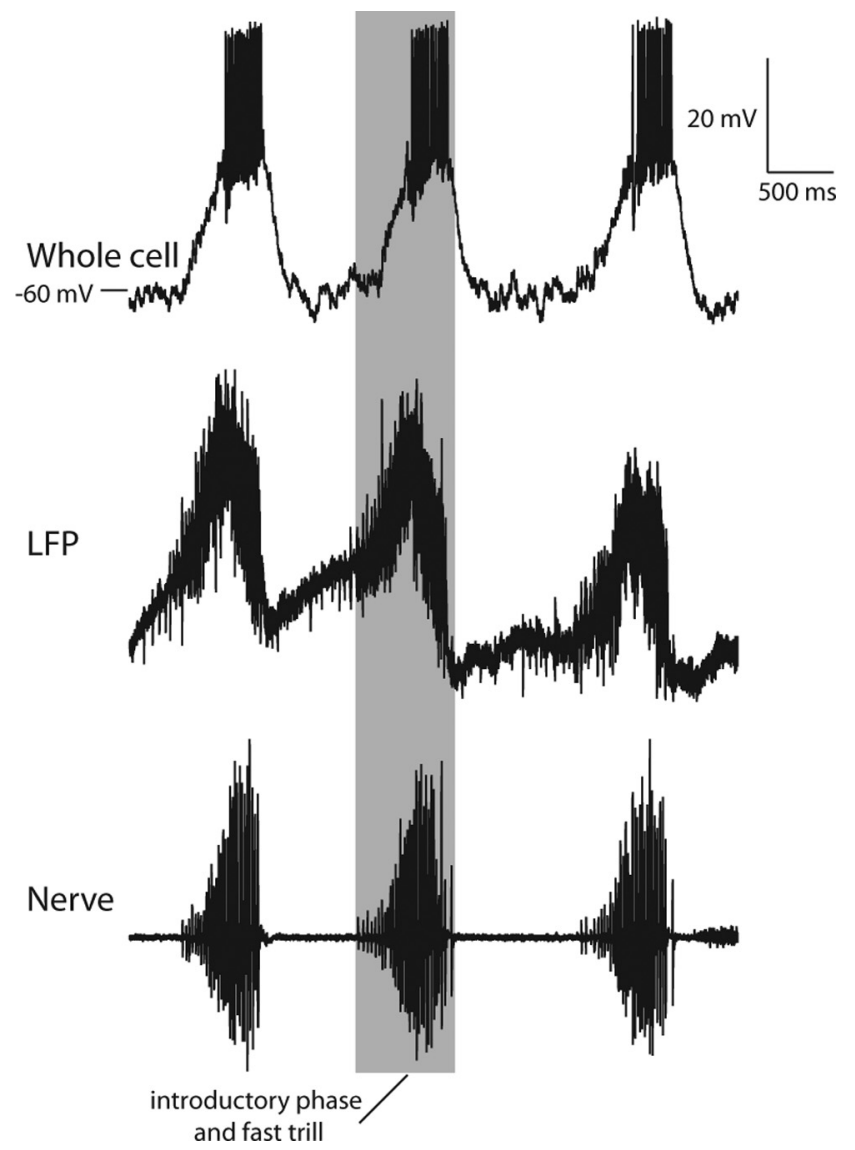

Figure 7. LLDs in FTNs coincide with LFP waves in DTAM. Example of whole-cell FTN activity (top trace) in which LFP activity was recorded from the contralateral DTAM (middle trace). In this recording, the fictive advertisement calls only show fast-trill and lack slow trills (as sometimes seen in vivo). Note that the LLD of the FTN correlates with both the fast trill on the nerve, and a baseline wave in the LFP recording.

We first tested whether NMDAR activation was necessary for LLDs as it is for LFP waves. Application of 5-HT to brains preincubated with $50 \mu \mathrm{M}$ APV prevents normal fictive calls in male brains as we have shown previously. Instead, we observed slow, low-amplitude CAPs that resemble those seen during introductory phase (Fig. 8, compare $A$ and $B$, nerve recordings). In the presence of APV, FTNs still received synaptic inputs correlated with introductory-phase CAPs but never produced LLDs or action potentials at fast-trill rates $(n=4$; Fig. $8 B)$. The result is consistent with the notion that the activation of NMDAR elicits LLDs, which in turn promotes the transition from introductory phase to fast trill.

We next tested whether NMDARs were expressed by FTNs themselves, or whether their activation occurred elsewhere in the circuit. To this end, we identified FTNs during fictive vocalizations (based on activity patterns in current-clamp mode), applied $1 \mu \mathrm{M}$ TTX to block voltage-dependent $\mathrm{Na}^{+}$channels (and spikemediated synaptic transmission), and then applied NMA (final concentration of 50, 75, or $100 \mu \mathrm{M}$ ). If FTNs express NMDARs, we reasoned that we may be able to record LLDs in response to NMA application in the presence of TTX, as has been shown in other neurons (Kiehn et al., 1996; MacLean et al., 1997; Prime et al., 1999). In 16 of 20 (80\%) FTNs exposed to NMA, we observed rhythmic repetitions of LLDs (Fig. 8D). The results indicate that activation of NMDA receptors expressed by the FTNs contributes to the initiation of the LLD. Furthermore, spontaneous termina- tion of LLDs in the absence of synaptic transmission indicates that one or more intrinsic outward currents must be expressed to repeatedly "turn off" the LLDs. Interestingly, the remaining four neurons (20\%) that did not show rhythmic LLDs became highly depolarized in response to NMA and never returned to the resting membrane potential. In these cells, the intrinsic outward current may not be present to terminate LLDs; alternatively, these cells may have higher expression of NMDARs, so that the NMAinduced currents prevented the outward current from overcoming the depolarizing drive. Together, the results indicate that (1) LLDs during fast trill likely require NMDAR activation, (2) NMDARs are expressed by the FTNs, (3) their activation elicits TTX-insensitive LLDs in the FTNs, and (4) most FTNs possess an outward current that can terminate NMDARdependent depolarizations.

Of 9 FTN neurons of 16 that showed stable repetition of LLDs for at least $15 \mathrm{~min}$ (see Materials and Methods), NMA-induced LLDs were $527 \pm 57 \mathrm{~ms}(n=9)$ in duration, repeated at an average rate of $1.0 \pm 0.08 \mathrm{~Hz}$ (range, $0.7-1.3 \mathrm{~Hz} ; n=9$ ). Considering that the duration and repetition rates of NMA-induced LLDs are similar to those of fast trills generated both in vivo and in vitro, LLDs may directly code for trill duration.

An important observation to be added here is that LFP recordings obtained from the contralateral DTAM showed no activity in response to NMA in the presence of TTX. The results suggest that, although each FTN shows rhythmic LLDs in response to NMA, these activities are not synchronized among cells and are therefore not recorded as an LFP wave. In this scenario, electrical coupling among FTNs, even if present, is not sufficient to synchronize the onset and offset of the LLDs across the FTN population.

Interestingly, in all nine neurons with stable and repetitive LLDs (induced by NMA in presence of TTX), we also observed STOs riding on top of LLDs (Fig. $8 E, F$ ). The average STO rate was very close to $60 \mathrm{~Hz}(60.8 \pm 9.6 ; \mu \pm \sigma)$. Thus, STOs are TTX insensitive and can be induced by NMDAR-dependent depolarizations. Unlike STOs induced in response to current injections, STOs on top of LLDs were observed at various membrane potentials $(-7$ to $-25 \mathrm{mV})$ across different neurons (membrane potentials were stable within individual neurons). When regression analyses were performed with average membrane potential as the independent, and average STO rates as the dependent variable, we found that variation in STO rates could not be explained by membrane potential (regression ANOVA, $F_{(1,7)}=0.53 ; p=$ 0.49 ). Therefore, while the activation of STO driving currents has a minimum voltage threshold, the rate of STOs seems to be voltage independent in FTNs.

In summary, FTNs express NMDARs that can initiate LLDs in response to presynaptic release of glutamate and an intrinsic capacity to terminate periodic LLDs, both of which may contribute to the timing of fast-trill onset and offset.

\section{Discussion}

\section{A single cell type may regulate both call rate and duration}

In response to vocalizations of gravid females, males produce a modified advertisement call with elongated fast trills (Tobias et al., 1998). Here, we showed that fast-trill duration varies within individuals while click rates remain constant, indicating that independent mechanisms regulate rate and duration. Surprisingly, we identified one cell type (FTNs) that may code both rate and duration via intrinsic resonance properties and NMDAR-induced LLDs. 

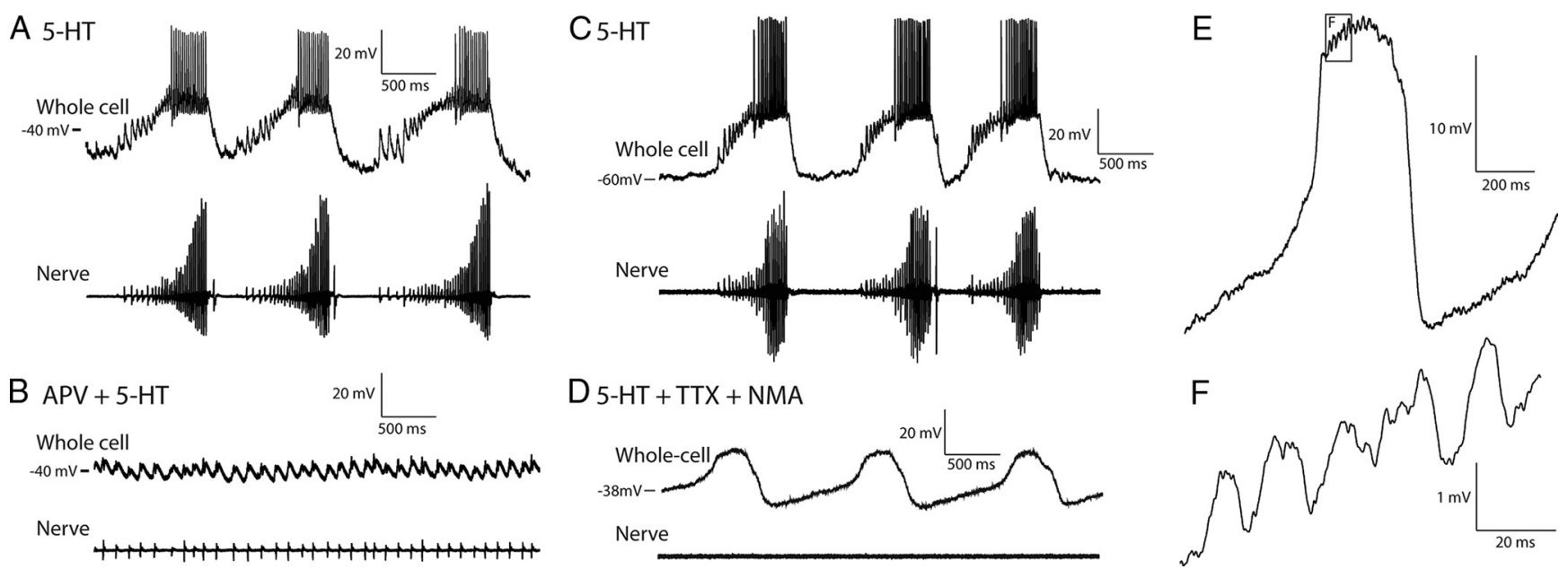

Figure 8. NMDAR activation in FTNs induces LLDs. A, Example FTN recording (top trace) during three fictive fast trills (bottom trace). $\boldsymbol{B}$, When APV was applied before a second 5-HT application, the FTN never produced LLDs or action potentials at fast-trill rates. Instead, this neuron received EPSPs during fictive introductory phase. C, Example FTN recording (top trace) during three fictive fast-trill renditions (bottom trace). $\boldsymbol{D}$, When the brain (during recording of neuron shown in $\boldsymbol{C}$ ) was treated with $1 \mu \mathrm{M} \mathrm{TTX} \mathrm{(to} \mathrm{eliminate} \mathrm{spike-mediated} \mathrm{synaptic} \mathrm{transmission)} \mathrm{and} \mathrm{then} \mathrm{exposed} \mathrm{to}$ the NMDAR agonist NMA, the FTN produced rhythmic pattern of LLDs. E, Example of a FTN producing a single LLD during NMDAR activation in the presence of TTX. Note that STOS appear in the membrane during the LLD. $\boldsymbol{F}$, Higher resolution view of STOs shown in $\boldsymbol{E}$.

\section{Mechanisms of coding call duration-role of NMDARs}

Because NMDAR antagonists block both LLDs and fast trills, LLDs may promote the transition from introductory phase to fast trill. Also, NMDAR activation in the presence of TTX typically induced rhythmic LLDs (rather than sustained depolarizations), indicating FTNs express an outward current that terminates LLDs (discussed in detail below). Therefore, FTNs may regulate fast-trill duration through the interplay of glutamatergic neurotransmission and an intrinsic outward current.

How does NMDAR activation induce rhythmic LLDs? In some neurons, NMDAR currents are the main charge carrier and induce membrane bistability (MacDonald et al., 1982; Sigvardt et al., 1985; Hochman et al., 1994; Kim and Chandler, 1995; Kiehn et al., 1996; Bonansco and Buño, 2003). In other neurons, calcium entry via NMDARs activates another conductance such as $\mathrm{Ca}^{2+}$-activated nonspecific cation current $I_{\mathrm{CAN}}$ to mediate LLDs (Lee and Tepper, 2007; Mrejeru et al., 2011). Calcium influx can also be involved in terminating LLDs by activating outward currents such as calcium-dependent potassium currents, $I_{\mathrm{K}(\mathrm{Ca})}(\mathrm{Hu}$ and Bourque, 1992; el Manira et al., 1994; Kim and Chandler, 1995; Shah and Haylett, 2002; Tahvildari et al., 2008). Identifying the currents underlying LLDs should reveal the cellular basis of fast-trill duration.

\section{Modulation of fast-trill duration without changing rate}

The neural mechanisms of fast-trill rate and duration are apparently distinct. One possible mechanism for elongating fast trills in sexually aroused males could be to change the kinetics of the outward current that terminates LLDs. With slowed current activation, LLDs would become elongated, producing a longer fast trill; assuming the outward current is not involved in generating STOs, trill rate would not change. Identification of this outward current will allow us to reveal the cellular basis of an arousaldependent behavioral change.

NMDAR-dependent membrane potential oscillations (similar to LLDs in this study) have been observed in many vertebrate neurons ( $\mathrm{Hu}$ and Bourque, 1992; Bonansco and Buño, 2003; Martell et al., 2010) including those controlling locomotion [lamprey (Wallén and Grillner, 1987); Xenopus tadpoles (Reith and Sillar, 1998; Li et al., 2010); rodents (Hochman et al.,
1994; Kiehn et al., 1996)]. The temporal resemblance between NMDAR-dependent oscillations and locomotor rhythms (Wallén and Grillner, 1987; Hochman et al., 1994; Li et al., 2010) suggested that this cellular mechanism may regulate locomotor rhythmicity. This prediction is supported in some systems [e.g., Xenopus tadpoles (Li et al., 2010)] but questioned in others [e.g., rodents (Cowley et al., 2005)]. The experimental accessibility of our preparation makes Xenopus vocalizations an excellent model for characterizing the cellular mechanisms of LLDs and their roles in rhythm generation.

\section{FTN rate coding}

How are FTNs tuned to $60 \mathrm{~Hz}$ ? STOs arise from a combination of passive membrane properties (e.g., membrane capacitance), resonant currents (e.g., the hyperpolarization-activated cation current $I_{\mathrm{h}}$, slow non-inactivating $\mathrm{K}^{+}$current $I_{\mathrm{M}}$, the $\mathrm{Ca}^{2+}$-activated $\mathrm{K}^{+}$current $I_{\mathrm{K}(\mathrm{Ca})}$, and a non-inactivating low-threshold 4-APsensitive current $I_{4-\mathrm{AP}}$ ), and amplifying currents (e.g., the persistent $\mathrm{Na}^{+}$current $I_{\mathrm{NaP}}$, current that flows through NMDAR channels $I_{\mathrm{NMDA}}$, and the dihydropyridine-sensitive high-threshold $\mathrm{Ca}^{2+}$ current $I_{\mathrm{L}}$ ) (for review, see Hutcheon and Yarom, 2000). Because STOs occur in the presence of TTX (Fig. $8 E, F$ ), we can rule out $I_{\mathrm{NaP}}$ involvement. Because brains from castrated male Xenopus generate fictive advertisement calls with slower fast trills (Zornik and Yamaguchi, 2011), identifying the cellular properties that underlie STOs will reveal how androgen modifies behavior of Xenopus by tuning oscillatory properties of a premotor neuron.

Although FTNs receive rhythmic synaptic inputs, they arrive after spikes, indicating that the synaptic inputs do not dictate FTN spike timing. Instead, synaptic inputs may represent an efferent copy of motor activity, and function to entrain the intrinsic STOs. We suggest that the interplay of intrinsic tuning and synaptic entrainment are the major fast-trill rate determinants. Cellular coding of a rhythmic motor program has been demonstrated in fish, in which vocal pacemaker neurons exhibit intrinsic STOs corresponding with call rates (Chagnaud et al., 2011). In other systems, STOs influencing the population rhythm are also prevalent. For example, STOs in cortical, thalamic, and olfactory neurons in mammals are thought to underlie EEG gamma rhythms (25-80 Hz) (Llinás et al., 1991; Chen and Shep- 
herd, 1997; Pedroarena and Llinás, 1997) that may underlie cognitive functions (Tallon-Baudry et al., 1998; Engel and Singer, 2001; Fries et al., 2001; Llinás et al., 2007), suggesting a causal relationship between STOs and neural rhythms.

For FTNs to dictate rhythmic motor output, FTN spikes must be synchronized. Neural correlates of fast trills (fast phasic activity in LFP recordings) in DTAM are abolished after transecting connections with n.IX-X (Zornik et al., 2010). This indicates that, although individual FTNs may be tuned to fast-trill rates, the DTAM circuit cannot generate synchronized $60 \mathrm{~Hz}$ rhythms autonomously. Instead, the ascending projection from n.IX-X may synchronize FTNs by providing an efferent copy of motor output. Thus, FTNs may continue to spike at fast-trill rates in the isolated DTAM, albeit asynchronously. The mixture of inhibitory and excitatory synaptic currents coinciding with the motor output recorded in this study may represent the efferent copy that synchronizes FTN spike timing.

An additional feature of FTNs is the tendency to produce spike doublets, which are most closely correlated with CAP amplitude. It has been suggested both theoretically and experimentally that spike doublets can enhance long-range neuronal synchronization in other systems (Traub et al., 1996). DTAM nuclei are separated bilaterally by $\sim 1 \mathrm{~mm}$, and n.IX-X is separated from DTAM by $\sim 3 \mathrm{~mm}$. Therefore, FTN spike doublets may also synchronize the activity of brainstem vocal nuclei.

\section{Evolution of vocal control circuits}

Similar to X. laevis, Plainfin Midshipman fish (Porichthys notatus) call durations are independent of rate (Brantley and Bass, 1994). In contrast to our findings, however, two neuron populations (in distinct nuclei) appear to code call rate and duration (Chagnaud et al., 2011). Despite this difference, it is noteworthy that rate and duration in both species appear to be regulated by the same cellular mechanisms, STOs and LLDs, respectively. Hindbrain circuits controlling vocal production in fishes, frogs, birds, and mammals may have derived from a common ancestor (Bass et al., 2008). Although intracellular recordings of hindbrain neurons have not been obtained in mammals or birds during vocalizations, it is possible that similar cellular mechanisms are involved in temporal coding.

Anatomical (Moreno and González, 2005; Zornik and Kelley, 2007) and physiological (Zornik and Kelley, 2008) evidence suggests that Xenopus DTAM is a homolog of the mammalian parabrachial nucleus (PB). PB in mammals is believed to coordinate respiratory and vocal activity (Jürgens, 2002), and is therefore ideal for controlling call duration in species with vocal mechanisms that rely on expiration. In bats, $\mathrm{PB}$ can influence the duration of expiration and calling (Smotherman et al., 2006). Although the Xenopus vocalizations are independent of respiration, the vocal mechanisms likely derived from ancestral species that relied on respiration during calling. The fact that some DTAM neurons are active during breathing or calling (although never both) supports our hypothesis that DTAM is a PB homolog. We suggest that regulation of call duration by $\mathrm{PB}$ may be conserved throughout the tetrapods.

\section{References}

Ashmore RC, Wild JM, Schmidt MF (2005) Brainstem and forebrain contributions to the generation of learned motor behaviors for song. J Neurosci 25:8543-8554.

Bass AH, Marchaterre MA, Baker R (1994) Vocal-acoustic pathways in a teleost fish. J Neurosci 14:4025-4039.

Bass AH, Gilland EH, Baker R (2008) Evolutionary origins for social vocalization in a vertebrate hindbrain-spinal compartment. Science 321:417-421.
Bonansco C, Buño W (2003) Cellular mechanisms underlying the rhythmic bursts induced by NMDA microiontophoresis at the apical dendrites of CA1 pyramidal neurons. Hippocampus 13:150-163.

Brantley RK, Bass AH (1994) Alternative male spawning tactics and acoustic signals in the Plainfin Midshipman fish Porchythys notatus Girard (Teleostei, Batrachoididae). Ethology 96:213-232.

Chagnaud BP, Baker R, Bass AH (2011) Vocalization frequency and duration are coded in separate hindbrain nuclei. Nat Commun 2:346.

Chen WR, Shepherd GM (1997) Membrane and synaptic properties of mitral cells in slices of rat olfactory bulb. Brain Res 745:189-196.

Cowley KC, Zaporozhets E, Maclean JN, Schmidt BJ (2005) Is NMDA receptor activation essential for the production of locomotor-like activity in the neonatal rat spinal cord? J Neurophysiol 94:3805-3814.

el Manira A, Tegnér J, Grillner S (1994) Calcium-dependent potassium channels play a critical role for burst termination in the locomotor network in lamprey. J Neurophysiol 72:1852-1861.

Engel AK, Singer W (2001) Temporal binding and the neural correlates of sensory awareness. Trends Cogn Sci 5:16-25.

Fries P, Reynolds JH, Rorie AE, Desimone R (2001) Modulation of oscillatory neuronal synchronization by selective visual attention. Science 291:1560-1563.

Hochman S, Jordan LM, Schmidt BJ (1994) TTX-resistant NMDA receptor-mediated voltage oscillations in mammalian lumbar motoneurons. J Neurophysiol 72:2559-2562.

Hu B, Bourque CW (1992) NMDA receptor-mediated rhythmic bursting activity in rat supraoptic nucleus neurones in vitro. J Physiol 458:667-687.

Hutcheon B, Yarom Y (2000) Resonance, oscillation and the intrinsic frequency preferences of neurons. Trends Neurosci 23:216-222.

Jürgens U (2002) Neural pathways underlying vocal control. Neurosci Biobehav Rev 26:235-258.

Kiehn O, Johnson BR, Raastad M (1996) Plateau properties in mammalian spinal interneurons during transmitter-induced locomotor activity. Neuroscience 75:263-273.

Kim YI, Chandler SH (1995) NMDA-induced burst discharge in guinea pig trigeminal motoneurons in vitro. J Neurophysiol 74:334-346.

Kubke MF, Yazaki-Sugiyama Y, Mooney R, Wild JM (2005) Physiology of neuronal subtypes in the respiratory-vocal integration nucleus retroamigualis of the male zebra finch. J Neurophysiol 94:2379-2390.

Lee CR, Tepper JM (2007) A calcium-activated nonselective cation conductance underlies the plateau potential in rat substantia nigra GABAergic neurons. J Neurosci 27:6531-6541.

Li WC, Roberts A, Soffe SR (2010) Specific brainstem neurons switch each other into pacemaker mode to drive movement by activating NMDA receptors. J Neurosci 30:16609-16620.

Llinás RR, Grace AA, Yarom Y (1991) In vitro neurons in mammalian cortical layer 4 exhibit intrinsic oscillatory activity in the $10-$ to $50-\mathrm{Hz}$ frequency range. Proc Natl Acad Sci U S A 88:897-901.

Llinás RR, Choi S, Urbano FJ, Shin HS (2007) Gamma-band deficiency and abnormal thalamocortical activity in P/Q-type channel mutant mice. Proc Natl Acad Sci U S A 104:17819-17824.

MacDonald JF, Porietis AV, Wojtowicz JM (1982) L-Aspartic acid induces a region of negative slope conductance in the current-voltage relationship of cultured spinal cord neurons. Brain Res 237:248-253.

MacLean JN, Schmidt BJ, Hochman S (1997) NMDA receptor activation triggers voltage oscillations, plateau potentials and bursting in neonatal rat lumbar motoneurons in vitro. Eur J Neurosci 9:2702-2711.

Martell A, Dwyer J, Koch H, Zanella S, Kohrman M, Frim D, Ramirez JM, van Drongelen W (2010) N-Methyl-D-aspartate-induced oscillatory properties in neocortical pyramidal neurons from patients with epilepsy. J Clin Neurophysiol 27:398-405.

Moreno N, González A (2005) Central amygdala in anuran amphibians: neurochemical organization and connectivity. J Comp Neurol 489:69-91.

Mrejeru A, Wei A, Ramirez JM (2011) Calcium-activated non-selective cation currents are involved in generation of tonic and bursting activity in dopamine neurons of the substantia nigra pars compacta. J Physiol 589:2497-2514

Nikundiwe AM, Nieuwenhuys R (1983) The cell masses in the brainstem of the South African clawed frog Xenopus laevis: a topographical and topological analysis. J Comp Neurol 213:199-219.

Pedroarena C, Llinás R (1997) Dendritic calcium conductances generate 
high-frequency oscillation in thalamocortical neurons. Proc Natl Acad Sci U S A 94:724-728.

Prime L, Pichon Y, Moore LE (1999) N-Methyl-D-aspartate-induced oscillations in whole cell clamped neurons from the isolated spinal cord of Xenopus laevis embryos. J Neurophysiol 82:1069-1073.

Reith CA, Sillar KT (1998) A role for slow NMDA receptor-mediated, intrinsic neuronal oscillations in the control of fast fictive swimming in Xenopus laevis larvae. Eur J Neurosci 10:1329-1340.

Remage-Healey L, Bass AH (2004) Rapid, hierarchical modulation of vocal patterning by steroid hormones. J Neurosci 24:5892-5900.

Rhodes HJ, Yu HJ, Yamaguchi A (2007) Xenopus vocalizations are controlled by a sexually differentiated hindbrain central pattern generator. J Neurosci 27:1485-1497.

Schmidt RS (1992) Neural correlates of frog calling: production by two semi-independent generators. Behav Brain Res 50:17-30.

Shah MM, Haylett DG (2002) $\mathrm{K}^{+}$currents generated by NMDA receptor activation in rat hippocampal pyramidal neurons. J Neurophysiol 87:2983-2989.

Sigvardt KA, Grillner S, Wallén P, Van Dongen PA (1985) Activation of NMDA receptors elicits fictive locomotion and bistable membrane properties in the lamprey spinal cord. Brain Res 336:390-395.

Smotherman M, Kobayasi K, Ma J, Zhang S, Metzner W (2006) A mechanism for vocal-respiratory coupling in the mammalian parabrachial nucleus. J Neurosci 26:4860-4869.

Subramanian HH, Holstege G (2009) The nucleus retroambiguus control of respiration. J Neurosci 29:3824-3832.

Tahvildari B, Alonso AA, Bourque CW (2008) Ionic basis of ON and OFF persistent activity in layer III lateral entorhinal cortical principal neurons. J Neurophysiol 99:2006-2011.

Tallon-Baudry C, Bertrand O, Peronnet F, Pernier J (1998) Induced gamma-band activity during the delay of a visual short-term memory task in humans. J Neurosci 18:4244-4254.

Tobias ML, Viswanathan SS, Kelley DB (1998) Rapping, a female receptive call, initiates male-female duets in the South African clawed frog. Proc Natl Acad Sci U S A 95:1870-1875.

Traub RD, Whittington MA, Stanford IM, Jefferys JG (1996) A mechanism for generation of long-range synchronous fast oscillations in the cortex. Nature 383:621-624.

Wallén P, Grillner S (1987) N-Methyl-D-aspartate receptor-induced, inherent oscillatory activity in neurons active during fictive locomotion in the lamprey. J Neurosci 7:2745-2755.

Wild JM, Kubke MF, Mooney R (2009) Avian nucleus retroambigualis: cell types and projections to other respiratory-vocal nuclei in the brain of the zebra finch (Taeniopygia guttata). J Comp Neurol 512:768-783.

Zornik E, Kelley DB (2007) Breathing and calling: neuronal networks in the Xenopus laevis hindbrain. J Comp Neurol 501:303-315.

Zornik E, Kelley DB (2008) Regulation of respiratory and vocal motor pools in the isolated brain of Xenopus laevis. J Neurosci 28:612-621.

Zornik E, Yamaguchi A (2011) Vocal pathway degradation in gonadectomized Xenopus laevis adults. J Neurophysiol 105:601-614.

Zornik E, Katzen AW, Rhodes HJ, Yamaguchi A (2010) NMDARdependent control of call duration in Xenopus laevis. J Neurophysiol 103: $3501-3515$. 